\title{
LA PLACE PUBLIQUE DANS LE ROMAN DE LAHBIB CHEBBI: LA FÊLURE
}

Por

ABDEL-HAKIM GAFSI

En tant qu'archéologue, je me suis proposé de dégager et de saisir, quelques traits spécifiques de la place publique dans la médina de Tunis, à partir de ce roman.

II ne s'agit pas, bien entendu, d'aborder la question de la place publique en tant qu'unité architecturale de base dans un tissu urbain moderne, mais de noter et de préciser quelques détails se rapportant à cette unité dans un cadre arabo-musulman (Tunis dans le roman) et relevés dans la fêlure.

Mais avant d'aborder la question, il est souhaitable de faire un rappel sur la structure de la médina de Tunis.

La médina ou la ville traditionnelle est de forme à peu prés ovale; elle est flanquée au Nord et au Sud de deux faubourgs: Bab Souika et Bab Dzira. La médina est aussi traversée du Nord au Sud et d'Est en Ouest par deux axes de circulation se coupant perpendiculairement au niveau des souks et de la Grande Mosquée (Zitouna).

A partir de ces grands axes rayonnent des parcours secondaires comprenant les differents quartiers, avec des ruelles enchêvetrées, ressemblant à des labyrinthes infinis, mais aussi des espaces publics, généralement le résultat de la jonction de ces deux axes avec les parcours secondaires.

Bien entendu, il n'existe pas de places publiques structurées sur le modèle grec ou romain (agora et forum distingués par lé régularité, l'harmonie et l'alignement), mais il s'agit plutôt de placettes que nous rencontrons sur les differents parcours de la médina de Tunis et qui sont en définitive un cadre conjoncturel et non structurel.

Par ailleurs, à l'Est de Tunis musulmane ou médina s'étend la cité européenne ou la Tunis «française» avec un quadrillage rigoureux de ses longues artères. Cette 
nouvelle cité, extra-muros, s'etait développé hors de la Porte de la Mer, dès le XVIII e siècle.

Cette petite analyse nous a permis de constater que deux Tunis s'observent et dont les frontières restent trés nettes. Par conséquent, les deux conservent une personnalité et un modèle de vie diamétralement opposés.

Cet espace rapidement évoqué servira de base et de point de départ à ce commentaire:

\section{1.- La place: domaine populaire par excellence.}

Reflet de l'organisation sociale, projection spatiale des structures sociales et vision de l'homme dans l'espace aménagé, la place publique est un espace intégré sur le plan morphologique comme sur le plan social. C'est un corps vivant; c'est le coeur palpitant de toute la cité. Lieu des actions simultanées: cloisonnement et ouverture, immobilité et mobilité... un thèatre ambulant: lieu de représentation, de divertissement, de hiérarchie. La place n'est qu'une conscience collective: «Sur la petite place, les hommes etaient réunis autour d'Ahmed. Je restai à l'écart aucun mouvement, à part Touhami qui servait, de temps en temps, sans hate, un café à un client. Pour l'eau, il n'y avait pas à se déranger, une jarre etait placée dans un coin du café: les clients se levaient et se servaient eux mêmes. La vie sur cette place semble avoir adopté un rythme au ralenti. Le cafetier et son serveur sont aussi interéssés par les histoires d'Ahmed que leurs clients. Le groupe est assis à même le sol et les bancs sont occupés par les vieillards» ( $P$ 44).

Les habitants sont liés à la place par un genre de contrat tacite; l'euphorie qui en résulte est le symbole de l'unité retrouvée, de l'emancipation. La place n'est en définitive que la vision concrète de l'existence humaine: «Mais peu importe, les habitants de notre capitale, mús par un instinct de joie indémontable, reviennent périodiquement à la place de la Kasba cueillir des émotions et des plaisirs, et ou oublier la monotonie quotidienne, le lot de toutes les ville ( $P$ 56).

Carrefour, voie de pénétration, lieu de convergence, de confluence, de rencontre, de rapprochement, d'animation diurne contrastant avec le vide nocturne, la place est une charge des multiples visages. Elle est donc le lieu de diversité, de multiplicité, de disparité, de contradiction, de défauts, de qualités, de libertés et même de dérapage. Coeur de la parole innocente, naïve mais aussi réfléchie, creuset des actes dramatiques ou comiques, la place est un objet vivant qui fascine par son ambiguité:

«Durant trois jours, c'est la joie qui investit la place avec ses cortèges de personnages pittoresques, diseuses de bonne aventure, conteurs, musiciens et lutteurs. Et chacun à sa façon, harangue la foule excitée que la fin du Ramadan ramène à une religiosité moins rigoureuse et un laisser aller bon enfant» ( $P 56$ ).

Objet de convoitise, lieu de servitude, de domination, de contrôle, projection des boulversements, changements, transformations, la place constitue une base de souvenirs; elle incarne et symbolise, par conséquent, malheurs et joies populaires. «La premiére fois, c'etait sur une pierre, juste au milieu de la place centrale de notre capitale. Les gardes, seuls occupants des lieux, jouaient aux cartes. La place, ainsi vidée de son monde, avait un aspect sinistre et je me suis mis à penser à ses moments. 
de forte animation. Je crois que c'est surtout durant les jours de fête qu'elle communique aux habitants un visage rassurant» ( $P$ 55).

\section{2.- Place et pouvoir}

La place n'est pas un espace neutre puisqu'elle incarne à la fois des idées de voisinage mais aussi de clientèle et de hiérarchie. De ce fait elle etablit un système complèxe de relations et de communications. Il ne s'agit pas donc ni d'un espace clos, ni d'un espace neutre. Intermédiaire entre la société et le pouvoir, la place est une caisse de résonance donc un espace privilégié pour le pouvoir: «ll est vrai que des débauches et des rixes viennent de temps à autre donner à la place un supplément d'émotion qure nos chouyoukhs, périodiquement, dénoncent à travers des dissertation qu'ils envoient au Maître de la Ville» ( $P$ 56).

Foyer de décision mais aussi de dédoublement, la place est le théatre de manifestations collectives et de croyances magico-religieuses. Elle est donc une balance dont les plateaux sont toujours en deséquilibre. Source de conflit la place publique demeure un foyer de tension perpetuelle:

«Un rassemblement occupait la place, fief habituel du conteur. Je pressentais les raisons de ce rassemblement et me demandais si le fournier n'avait pas dû se ressaisir à temps: entre lui et le mouharrek du quartier, je sentais qu'il n'y avait aucune possibilité d'ententes (P 107).

Image, vitrine, miroir de ce conflit, la place restera toujours un enjeu: Incarnation des contraintes des normes d'un pouvoir mais aussi représentation réelle ou imaginaire d'une conception dramatique d'une réalité sociale:

«La seconde fois que je m'assis, j'étais sur la place populaire du faubourg. Elle etait animée avec un rien de reserve, probablement imposée par le choléra... Je pouvais m'intégrer aux groupes qui, assis à même le sol, discutaient. J'ai préféré m'asseoir à part et me laisser envahir des souvenirs. Cette place a, de tout temps, été un lieu apprécié par les classes populaires, mais le pouvoir l'a aussi marquée du sceau de sa repression. C'est sur cette place qui avaient lieu les exécutions capitales» (P 59).

Lieu d'interférences, la place peut produire des contrastes, des differences des discontinuités, des anomalies, des conflits. Résultante de trois forces opposées: pouvoir, adultes, enfants, cet espace reste un berceau d'un acte inachevé «Jusqu'à un certain point, quant les adultes commençaient à vider la place, en jetant parfois des pierres au bourreau, nous étions, nous les enfants déçus» (P 61).

Lieu de sociabilité, d'échange et d'ouverture donc de rapports pacifique la place est le cadre oû se renouent l'alliance profonde, l'harmonie, la cohésion, l'entente, la similitude et la communion:

«Je débouchai sur la place aux moutons. Les hommes, par petits groupes, calmement assis, profitaient du soleil matinal» (P 185).

\section{3.- La place: lieu de l'inconnu}

Mobile ou immobile, connu ou inconnu, limité ou illimié, la place est un pivot autour duquel s'enchaînent les hommes: 
«En quittant SI Aissa, je ne suis pas rentré chez mol. Je ne suis pas non plus resté sur la place pour écouter Ahmed finir son conte. J'ai passé le reste de la matinée à errer» (P 54).

La place est un réseau fait d'enclos, d'interdiction, d'ouverture, d'accéssibilité de fermeture et d'exclusion donc un contraste inconnu de vide et de plein, d'espace libre et de labyrinthe, d'ouvert et de fermé, du privé et du public, de la liberté et de la servitude:

«Salah Jeridi, emportant l'argent de la victime, s'engagea dans les ruelles qui partent de la place Bab Suika et crut ainsi m'échappers ( $P$ 212).

La juxtaposition de plusieurs individus, l'entassement ou la concentration de la population dans cet espace relativement restreint offrent des ambiguités: solidarité sociale ou discipline collective, tolérance mutuelle ou diversité sociale et économique, émancipation sociale ou prestige personnel, c'est à dire des lignes de démarcation assez flous malgré une fluidité apparente. Donc la hiérachie, l'ordre conventionnel, la conception collective des rapports entre individus ou symbole et anonymat demeurent les dimensions de la recherche de l'équilibre dans cet espace.

«Je pouvais déboucher directement sur la place, mais Ahmed le conteur me verrait et se tairait par respect envers un cheikh, ou bien contourner tout le bloc d'habitations et de commerce, de façon à me ménager un moment oû je pourrais écouter à ma guise ce qu'il raconte» ( $P$ 42).

Lieu du sacré et du profane, du permis et de l'interdit, du dialogue, de la coexistence du réel et de l'imaginaire: "Notre rue, oû campe le Kouttab de si Lkhdiri, débouche sur une petite place de Ahmed, conteur populaire du coin» ( $P$ 15).

Lieu d'intégration des espaces d'intimité, individuels et familiaux, lieu de médécine donc d'espoir, de création et d'imagination:

«Sur cette place, outre le café oû campe majestueusement Ahmed le conteur, SI Aissa, de mémoire d'homme, a toujours été vu assis devant sa boutique "aux herbes médicinales"» ( $\mathrm{P}$ 16).

\section{4.- Place et colonisation}

La transplantation d'une civilisation étrangère, le changement des réalités, le déplacement des priorités qui s'opèrent à Tunis et au détriment de la médina entrainent un transfert, une migration, une poussée vers un autre espace plus approprié aux nouvelles conditions. L'incompatibilité de la médina, son inadaptation, résultats de la redistribution des activités aussi bien économique que sociale, se traduit inévitablement d'une modification de l'implantation géographique et l'eclipse de la médina. Une nouvelle dichotomie s'instaure. Le centre devient péréphérie et vice versa.

Cette nouvelle configuration, héritage de deux civilisations opposées, représente sur le terrain la juxtaposition de deux communautés differentes, musulmane et chrétienne, arabe et européenne. La place nourrit donc le cloisonnement de groupes opposés et le fractionnement. De la place surgit donc un nouveau système de privilége.

Bien entendu de ces transformations ont surgi de nouveaux rapports et de nouveaux déséquilibres, déséquilibres qui résultent d'une confrontation inégale. La pla- 
ce devient donc le théatre de deux modèles de civilisation qui s'affrontent et s'entremêlent en même temps:

"Les musulmans ne s'aventurent pas plus loin que la place de la Bourse et donnent l'impréssion de suivre cette déambulation toute europèenne sur la Promenade de la Marine» (P 170).

«Sur cette place de la Bourse, déambulant lentement devant moi Monsieur Pierre semblait venir d'un autre monde...» (P 175).

Par conséquent, cette nouvelle place engendre la frustration, la résignation, le pessémisme, le deséspoir, l'évasion dans le rêve, peut-être, mais surtout le mépris des valeurs morales et les traditions.

«En arrivant au niveau de la rue el Qramed, femmes et enfants virèrent à droite et commencèrent à suivre le flot d'habitants d'autres quartiers qui descendaien la rue en direction de la place de la Bouse. Je n'avais plus besoin de les suivre. Je savais que mes compatriotes, délaissant leur médécine, leur croyance, dans l'inéluctable, leur confiance dans les saints, se dirigeaient vers les lieux européens de consultation» (P 114).

La place est donc le témoin de l'accèptation résignée des formes de la culture dominante, résultat de l'illusion, du blocage, de la méfiance de l'ankylose «Mais sur cette place de la Bourse, la pensée de mes compatriotes reste trop prés de réalités pour se permettre de telles divagations» (P 172).

Solitude, anxiété, despersonnalisátion, repli sur soi même, telles sont les conséquences de ces nouvelles réalités. La place divient par conséquent le cadre idéal de la recherche de la paix, de l'harmonie et surtout l'identité:

«Je m'étire à longueur des journées et d'années dans mes rues, mes place et mes impasses» ( $P$ 136).

\section{Conclusion}

La place publique, chez Lahbib Chebbi, est dominée par le conteur populaire. II s'agit donc d'un cadre irréel et imaginaire car la place publique par excellence dans la medina est la mosquée, oû prend corps la communauté urbaine.

Lahbib Chebbi, a voulu, peut être, exprimer son rêve d'exalter, la médina qui l'a bien connu, par l'intermédiaire de la place publique, cadre idéal de l'imagination. De là faut-il rappeler que Lahbid Chebbi n'a pas cherché à saisir la nature des mutations ni les perspectives qu'elles ouvrent, ni les formes, les mouvements et les couleurs offerts par la médina. 